\title{
Fluidic gates simulated with lattice Boltzmann method under different Reynolds numbers
}

\author{
Michail-Antisthenis Tsompanas ${ }^{\mathrm{a}, *}$, Andrew Adamatzky ${ }^{\mathrm{a}}$ \\ ${ }^{a}$ Unconventional Computing Lab, Department of Computer Science and Creative Technologies, University of the West of \\ England, Bristol, UK
}

\begin{abstract}
Fluidic devices use fluid as a medium for information transfer and computation. Boolean values are represented by the presence of fluid jets in the input and output channels. Velocity of a fluid is one of the parameters determining Reynolds number of the flow. Reynolds number is a parameter that characterizes the behaviour of the flow: laminar, transient or turbulent. Using lattice Boltzmann method we study the behaviour of fluidic gates for various Reynolds numbers. On the designs of AND and oR gates we show the fluidic gates remain functional even for low Reynolds numbers, like 100. The gates designed can be cascaded into functional logical circuits.
\end{abstract}

Keywords: fluidic logic gates, Reynolds number, lattice Boltzmann method, multiple-relaxation-time

\section{Introduction}

Devices that employ dynamic phenomena of fluid flows to execute logic operations, sensing or controlling are defined as fluidic systems. These systems do not depend on mechanical moving parts or on electronic elements [1. Back in 1920, Tesla [2] has proposed the first fluidic device, practically working as a diode. Tesla's design is allowing the flow of liquid towards one direction with a minor resistance, whereas imposing an almost impassable barrier when flowing in the opposite direction. The designs of fluidic computational devices particularly flourished in 1950s and early 1960s with the aim to develop reliable devices without or with minimum electronics components $3,4,5$. Key principles of the functionality of a fluidic gate include laminar flow of a fluid, jet interaction, wall attachment and vortex effect. Notably, in 1963 Warren and Horton designed implementations of fluidic gates AND-NOT, AND and OR-NOR [6]. Recently interest to fluidic computing got reignited due to trends in future and emergent computing engineering, including IEEE initiative on rebooting computing [7, 8, 9, 10, and focus of the unconventional computing field on experimental prototyping of novel computing devices [11].

The fluidic devices designed in 1950s-1960s were reported as operating at high Reynolds numbers $(R e)$ characterizing the input flows, namely to operate with high speed jets. The fluidic devices, particularly fluidic amplifiers, are reported to have acceptable gains, thus, operating sufficiently, under fluid flows with Re of approximately 1000 [12]. This prevents miniaturization of the fluidic devices: definition of Re implies that for dimensions under a millimeter, the required velocity has to be so high that makes the devices impractical. This is why the research community shifted to devices utilizing fluid flows with low Reynolds numbers [13. However, the fact that it has been reported that even in flows with Re numbers lower than 100, bifurcations can be observed 14, indicates that fluidic devices could be functional with lower $R e$ values.

\footnotetext{
* Corresponding author

Email addresses: Antisthenis.Tsompanas@uwe.ac.uk (Michail-Antisthenis Tsompanas), Andrew.Adamatzky@uwe.ac.uk (Andrew Adamatzky)
} 
Some recent studies have shed some light into the functionality threshold of fluidic devices, like the bistable fluidic valve, through experimental methodologies [15]. Despite the successful growth of computational fluid dynamics (CFD) in recent decades, only a handful of studies were conducted, investigating further the characteristics of fluidic devices. For example, in [16] the switching operation in a fluidic amplifier was simulated via a commercial CFD program.

There is no accurate estimation of a critical $R e$ that would allow a correct functioning of fluidic devices. Moreover, it is not studied how all the outputs of these devices are rated when operated under sub-threshold Re. As a result, we aimed to classify the regimes of fluid flow for which fluidic gates are functioning properly. We simulate fluid dynamics using lattice Boltzmann method (LBM) because it offers the following advantages. The local version of a kinetic equation used allows for a straightforward implementation of boundary conditions and mapping simulated space to processors in parallel computers. Geometry of a simulated fluidic device can be changed without changing the meshing of the fluid domain.

\section{Methods}

We simulated fluid flow using the LBM [17, 18, 19, 20, 21, 22, where the fluid is represented in an orthogonal grid of nodes as particle distribution functions. This method studies ensembles of particles to derive macroscopic phenomena, thus the key concept is to alleviate the distance between micro-scale and macro-scale simulation techniques [23. LBM was proved to be a good alternative to other CFD methods [24. The key advantages that enhanced its applicability to many engineering problems [25, 26], are the straightforward manner to tackle challenging configurations (like flow through porous media or complex geometries), simplicity and ease of conversion of the algorithms implementing the method to be executed on parallel computing platforms [27, 28, 29, 30, 31, 32].

The two-dimensional and nine discrete velocity (D2Q9) lattice (Fig. 1) model with multiple relaxation times (MRT) [33, 34, 35] was used, as it is numerically stable for high Re numbers. The simulation comprises by the calculation of the lattice Boltzmann equation for all nodes in the grid for consequent time steps $(t)$, which derives the particle distribution functions. Lattice Boltzmann equation is defined as follows:

$$
f_{q}\left(\overrightarrow{x_{j}}+\overrightarrow{c_{q}} \delta t, t+\delta t\right)=f_{q}\left(\overrightarrow{x_{j}}, t\right)+\Omega_{q}\left(\overrightarrow{x_{j}}, t\right)
$$

where $f_{q}$ is the $q$-th component of the particle distribution functions vector, $\overrightarrow{x_{j}}$ is a location of the lattice node, $\overrightarrow{c_{q}}$ the $q$-th discrete velocity and $\Omega_{q}$ the $q$-th component of the collision operator, here $q=0, \ldots, 8$ for the D2Q9. The discrete velocities for the D2Q9 grid, depicted in Fig. 1, are given as:

$$
\overrightarrow{c_{q}}= \begin{cases}(0,0) & q=0 \\ ( \pm 1,0),(0, \pm 1) & q=1, \ldots, 4 \\ ( \pm 1, \pm 1) & q=5, \ldots, 8\end{cases}
$$

The local hydrodynamic macroscopic quantities of density and velocity are simply derived by summations:

$$
\rho=\sum_{q} f_{q} \quad \vec{u} \rho=\sum_{q} \overrightarrow{c_{q}} f_{q}
$$

While the collision operation in single relaxation time LBMs (like the well-established Bhatnagar-GrossKrook model 24]), happens in the velocity space, in the MRT model it is developed in the moment space. A vector of the nine moments is:

$$
\vec{m}=\left(\rho, e, \epsilon, j_{x}, q_{x}, j_{y}, q_{y}, p_{x x}, p_{x y}\right)^{T}
$$

where $\rho$ is the density, $e$ energy, $\epsilon$ energy-square, $j_{x}$ and $j_{y}$ momentum in $x$ - and $y$-dimension, $q_{x}$ and $q_{y}$ heat flow in $x$ - and $y$-dimension and $p_{x x}$ and $p_{x y}$ diagonal and off-diagonal stress.

The transformation from the velocity space and the particle distribution functions to the moments space (and vice versa) is achieved by: 


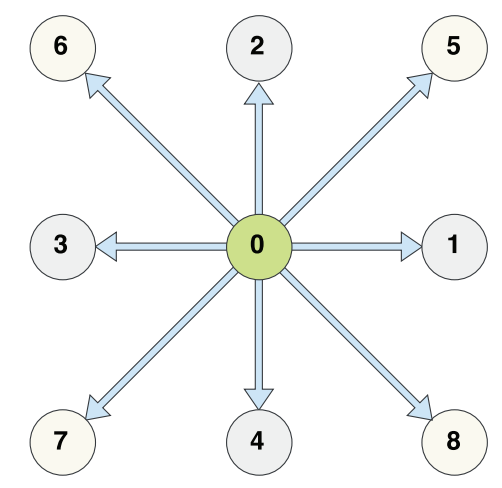

Figure 1: The two-dimensional and nine discrete velocity (D2Q9) lattice

$$
\begin{gathered}
\vec{m}=M \vec{f} \\
\vec{f}=M^{-1} \vec{m}
\end{gathered}
$$

For MRT, the collision operator $\Omega$ is approximated by a collision matrix $\widehat{S}$ as:

$$
\vec{\Omega}=-M^{-1} \widehat{S}\left(\vec{m}-\vec{m}^{e q}\right)
$$

where

$$
\widehat{S}=\operatorname{diag}\left(s_{0}, \ldots, s_{8}\right)
$$

where $s_{q}$ are the nine relaxation parameters set inside $(0,2)$. Here the parameters are chosen as $s_{0}=s_{2}=$ $s_{3}=s_{5}=1, s_{1}=s_{4}=s_{6}=1.2$ and $s_{7}=s_{9}=\omega$; where $\omega$ is the collision frequency and it is determined according to the simulated Re number for every case.

The transformation matrix is defined as:

$$
M=\left[\begin{array}{ccccccccc}
1 & 1 & 1 & 1 & 1 & 1 & 1 & 1 & 1 \\
-4 & -1 & -1 & -1 & -1 & 2 & 2 & 2 & 2 \\
4 & 2 & 2 & 2 & 2 & 1 & 1 & 1 & 1 \\
0 & 1 & 0 & -1 & 0 & 1 & -1 & -1 & 1 \\
0 & -2 & 0 & 2 & 0 & 1 & -1 & -1 & 1 \\
0 & 0 & 1 & 0 & -1 & 1 & 1 & -1 & -1 \\
0 & 0 & -2 & 0 & 2 & 1 & 1 & -1 & -1 \\
0 & 1 & -1 & 1 & -1 & 0 & 0 & 0 & 0 \\
0 & 0 & 0 & 0 & 0 & 1 & -1 & 1 & -1
\end{array}\right]
$$

The MRT method is described in detail in [33, 34, where also the definition of the equilibrium of moments $\left(\vec{m}^{e q}\right)$ for Eq. 6 can be found.

The boundary conditions in the simulated wall area is defined as halfway bounce-back boundary conditions, that have been proved to be second order accurate [36]. Moreover, the boundary conditions in the inlet of the simulated configurations are implemented as in 37. with a given and stable velocity in the $x$-axis.

The simulations were executed in MatLab R2016b and the actual fluidic gate geometry is replicated in a $100 \times 100$ grid. The nodes represent solid boundaries and free space for the fluid to flow. The solid boundaries are coloured in black and free space is white in the illustrations.

We studied two configurations of gates shown in Fig. 2. The geometry of the AND gate used in the simulations is shown in Fig. 2a, adopted from sketches presented in [38, 39]. The presence of fluid flow in 


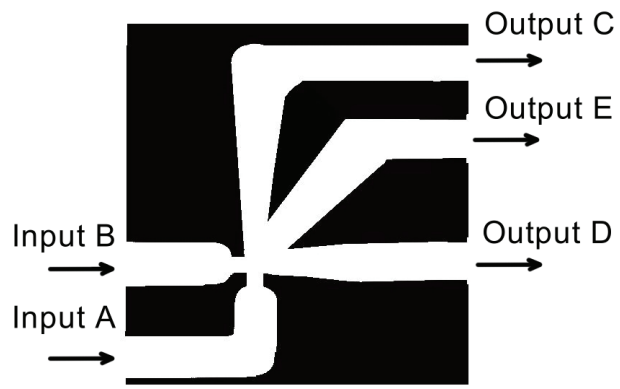

(a)

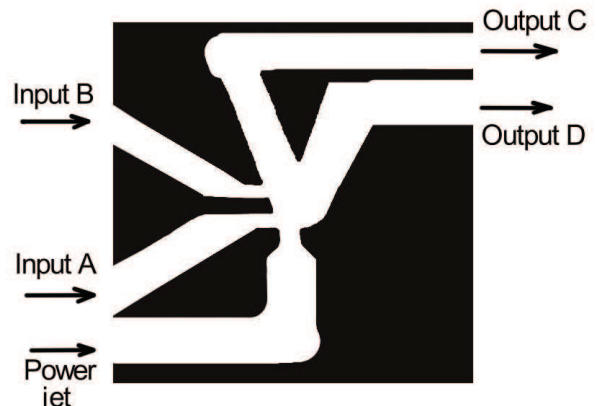

(b)

Figure 2: The configurations of the (a) AND and (b) or gates. Adopted from 38] 39]

output $\mathrm{C}$ represents the state "only A" $(A \bar{B})$, in output $\mathrm{E}$ the state "A AND B" $(A B)$, while in output D the state "only B" $(\bar{A} B)$. The geometry of the or gate is adopted from sketches proposed in 38,39 , 3nd illustrated in Fig. 2 2 b. The presence of fluid flow in output $\mathrm{C}$ is translated as the state "A NOR B" $(\overline{A+B})$, while in output D the state "A or B" $(A+B)$. Note here that the velocity of the outputs is presented in comparison with the velocity of the input; namely as a fraction between the output and the input velocity of the input that is activated for every case. Furthermore, for illustration and uniformity reasons, all input and output velocities are considered only in the $x$-axis direction.

\section{Results}

Both gates were tested with the simulation of flows in the range from 25 to $300 R e$ number. We considered pipe-like channels, so $R e$ number for a flow in a pipe is defined as: $R e=\frac{u \cdot D_{H}}{\nu}$, where $u$ is the mean velocity of the flow, $D_{H}$ is the hydraulic diameter of the pipe and $\nu$ is the kinematic viscosity of the fluid. The dimensions of these parameters have to be chosen in such a manner so as the Re number to be a dimensionless quantity. The output velocities of the flow were compared with the input ones to determine if the gates would function correctly. Note here that the exact values of the aforementioned parameters are not critical (within reasonable limits), as long as their ratio results in the targeted $R e$ numbers. Nonetheless, the $D_{H}$ parameter was considered a constant throughout all the different cases studied, in order to simulate a specific geometry with the same dimensions. Namely, it is set to $0.01 \mathrm{~m}$. Moreover, the kinematic viscosity $(\nu)$ of water was used at $20^{\circ} \mathrm{C}$ which is equal to $10^{-6} \mathrm{~m}^{2} / \mathrm{s}$. To derive the targeted $R e$ numbers the input velocity of the fluid is allocated within the range of 0.0025 to $0.03 \mathrm{~m} / \mathrm{s}$.

\subsection{AND gate}

The first case studied is when only input $\mathrm{A}$ is activated. Flow fields for $R e=50$ and $R e=350$ are illustrated in Fig. 3 and the output velocity versus $R e$ in Fig. 4 (a). Output $\mathrm{C}(C=A \bar{B})$ presents a comparable velocity with the one in the input for $R e \approx 100$ and higher. This is an indication of the proper functionality of the gate. What is noteworthy here, is that the output $\mathrm{E}$ has a small negative velocity, whereas in output D a higher negative velocity is present. This can be attributed to the fact that the Coanda effect [40, 41 takes place, thus, the ambient pressure of the jet exiting the nozzle of input A is low. This in turn causes the fluid to flow in a negative direction on the $x$-axis than the one defined as the output D. The aforementioned phenomenon can be observed in Fig. 3(c) and 3(d)] where the fluid is backtracking mainly from output $\mathrm{D}$ towards the output $\mathrm{C}$. On the other hand, when $R e=50$ the input jet is divided between outputs $\mathrm{C}$ and $\mathrm{E}$ as illustrated in Fig. $3(\mathrm{a})$ and $3(\mathrm{~b})$. The pressure contour for $R e=300$ is illustrated in Fig. 5(a) where the lower values of pressure can be observed around the output of nozzle A. 


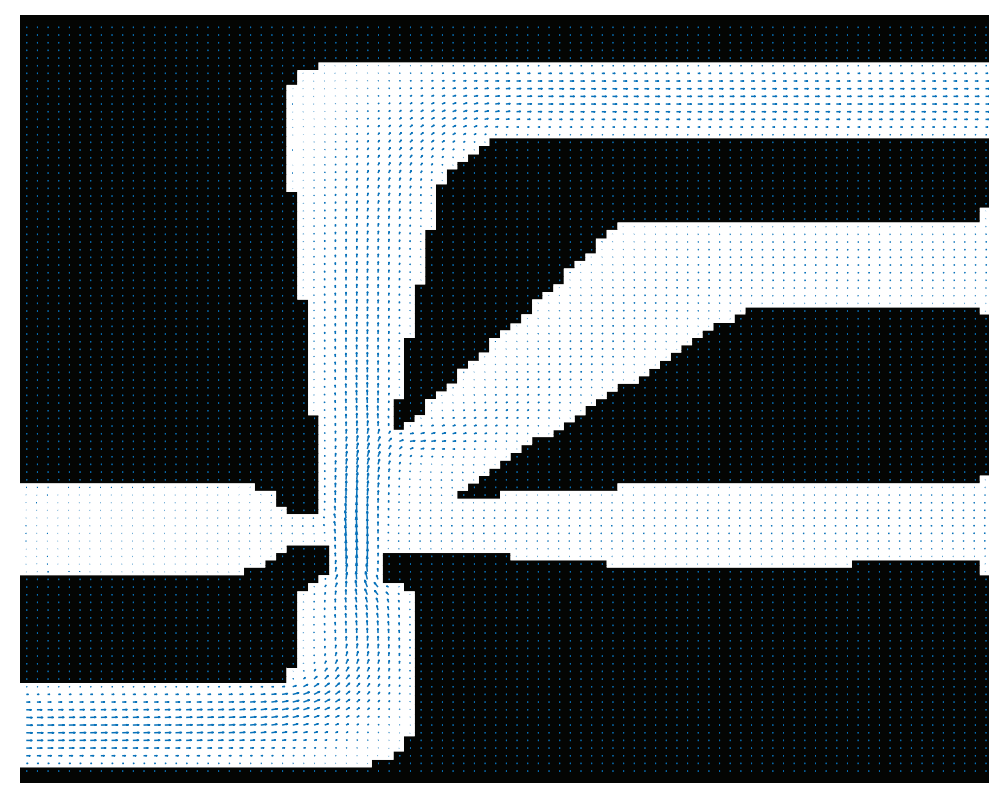

(a)

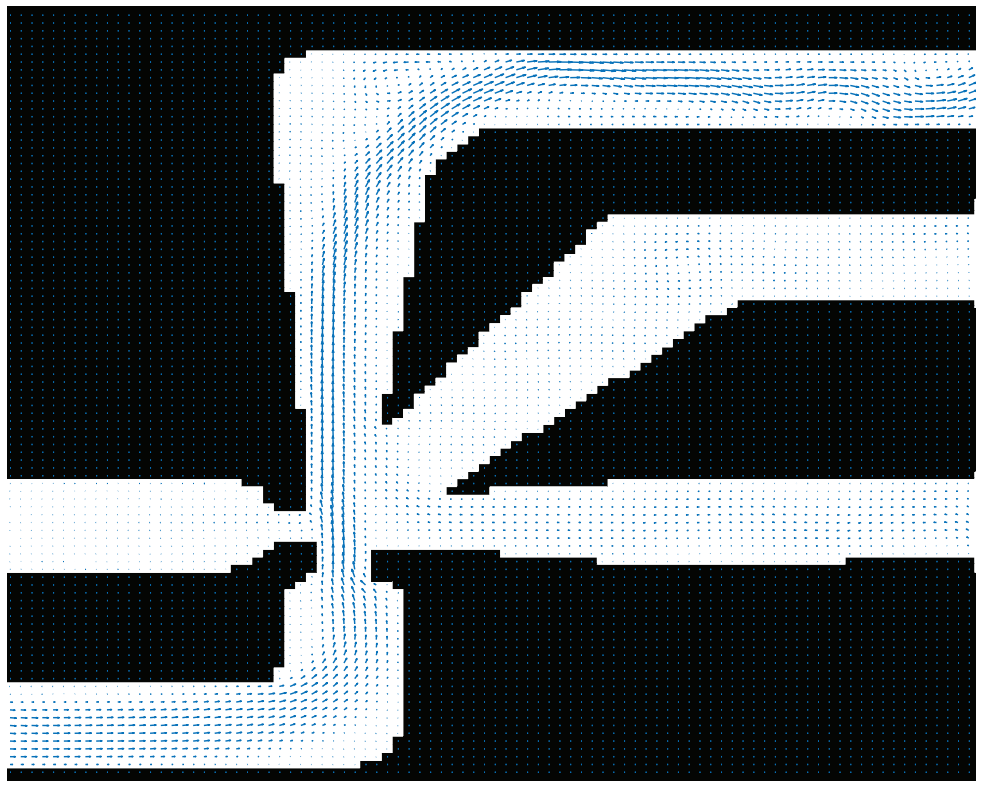

(c)

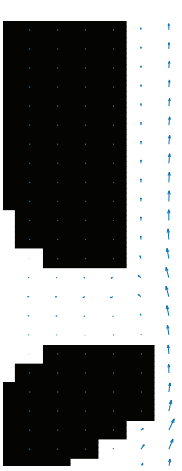

(b)
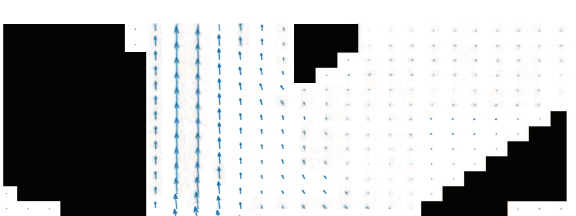

Figure 3: Flow field for AND gate (Fig. 2a) with input A activated for (ab) low, $R e=50$, and (cd) high, $R e=350$, Reynolds numbers. (ac) Overview of flow fields. (bd) Zoomed flow fields in the nozzle area. 


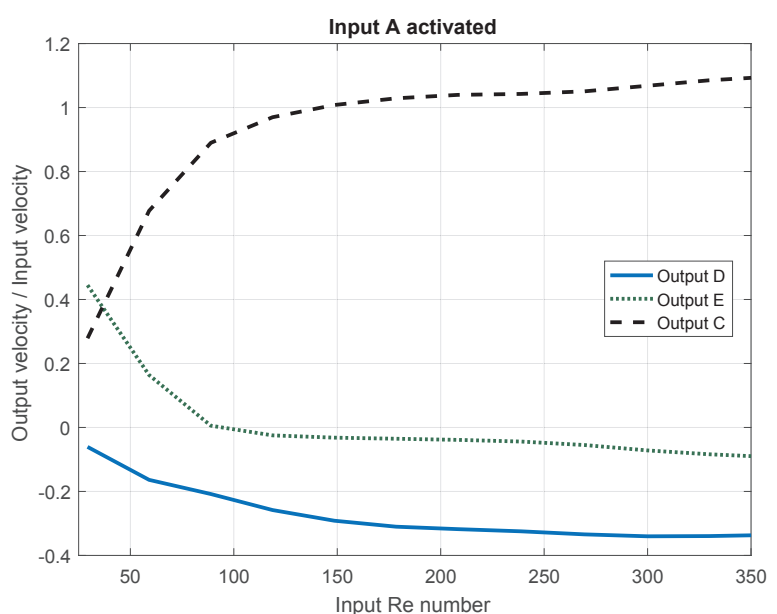

(a)

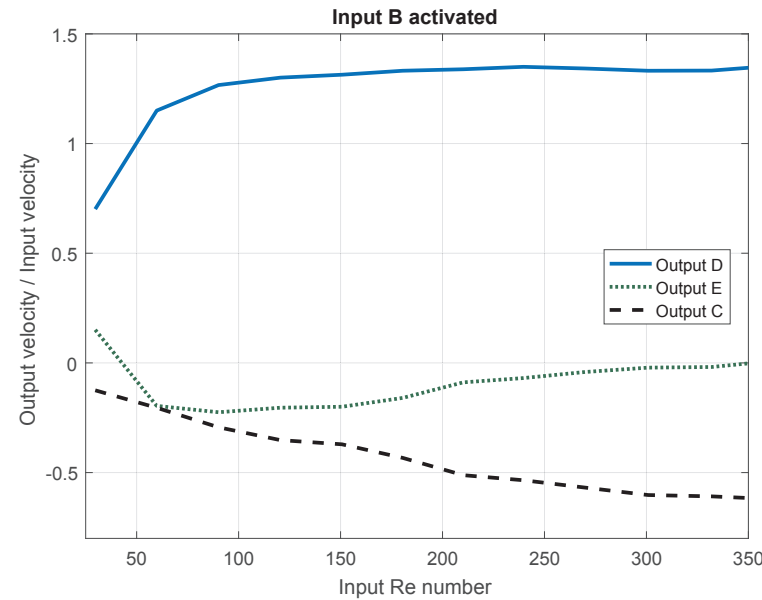

(b)

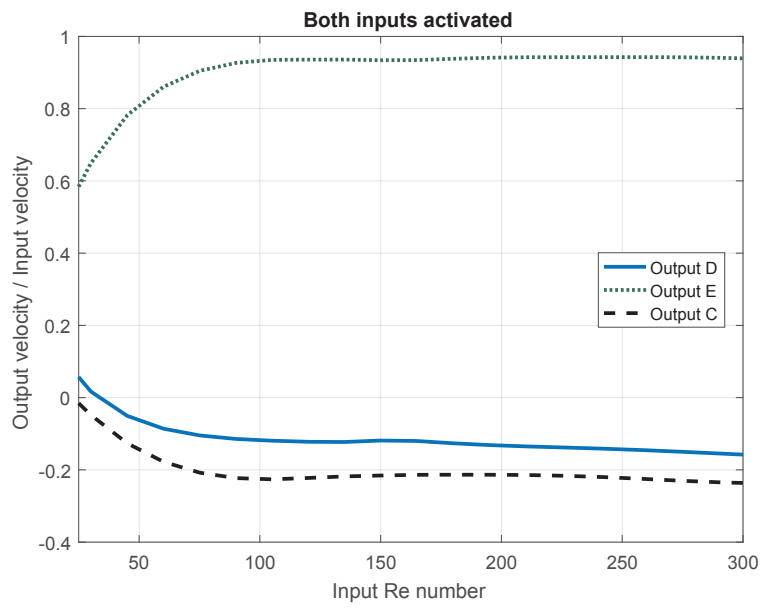

(c)

Figure 4: Relative velocity of fluid flow in output channels of AND gate (Fig. 2p) with (a) input A, (b) input B, (c) both inputs activated. 


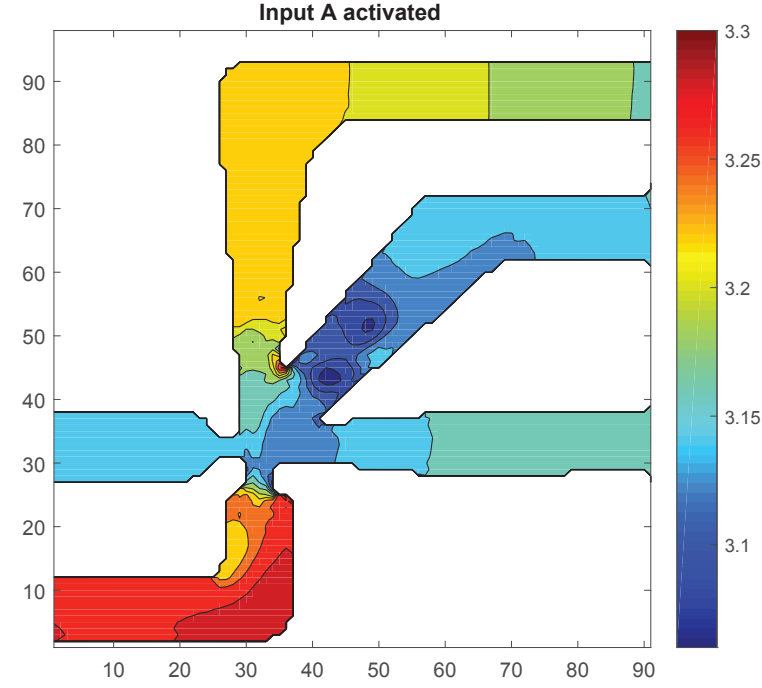

(a)



(b)

Figure 5: Pressure contour for $R e=300$ of AND gate, when the output jet is forming (a) for input A activated and (b) for both inputs activated.

Then, the case when only input $\mathrm{B}$ is activated is investigated and the outputs of the simulation are depicted in Fig. 4(b). Here, the functionality of the gate is correct and measurable for $R e \approx 50$ and higher. That statement is apparent from the relative velocity of output $\mathrm{D}(D=\bar{A} B)$. In a similar way, as the aforementioned, the Coanda effect is realized, hence the negative velocities in the other two outputs. Moreover, the higher relative output velocity can be explained by the shorter output path which, also, does not accommodate any breaks/change of direction (as the other outputs do).

When both inputs are activated the gate seems to produce an output with velocity at $80 \%$ of the average of the input velocities at output $\mathrm{E}(E=A B)$ for Re numbers of 50 and higher (Fig. 4 (c)). Once again, in the other outputs a negative velocity is apprehended due to the low pressure zones in the sides of the resulting jet after the interaction of the two input jets, as depicted in Fig. 5(b).

\subsection{OR gate}

When none of the inputs are activated in oR gate but the power jet is present, in output $\mathrm{C}(C=\overline{A+B})$ a flow is developed with $80 \%$ of the input velocity for input Re numbers of 100 and higher (Fig. 6a). On the other hand, because of the Coanda effect, there is a negative velocity in output D.

For all the other states of the inputs (only A, only B and both inputs), a flow in output $\mathrm{D}(D=A+B)$ must be observed. The simulation justifies this assumption, as realized in Fig. 6(b)(c)(d). Moreover, the effect that the lower pressure near the jet is clearly identified again, as output $\mathrm{C}$ develop flows with negative velocities (Fig. 66. Note here that in the case where input B is activated, because of the placement of the divider of outputs closer towards the nozzle of input B (compared with the nozzle of input A), the output is not that distinct as in the other cases. The output reaches $80 \%$ of the input velocity for Re numbers exceeding 250 .

Moreover, the flow fields for OR gate with input A activated for $R e=50$ and $R e=350$ are shown in Fig. 7 The interaction of input jets for low Re number and the formation of a vortex in the path towards output $\mathrm{C}$ can be observed in Fig. 7(a) and 7(a) Moreover, the higher output velocity of output $\mathrm{D}$ and the negative velocity in output $\mathrm{C}$ for a high $R e$ number can be noticed in Fig. $7(\mathrm{c})$ and $7(\mathrm{~d})$, As the resulting jet is diverted towards the right wall, the pressure on the other side of the jet is lower and, thus, output $\mathrm{C}$ is developing a negative velocity. 


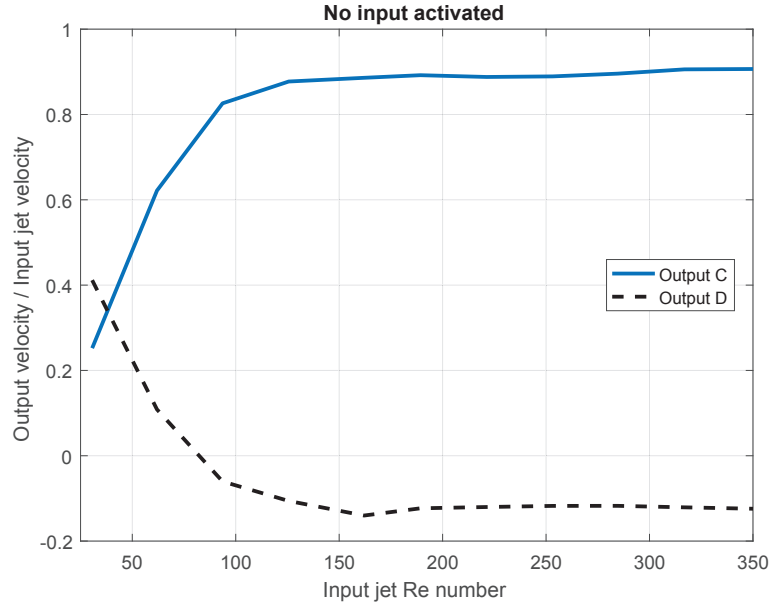

(a)

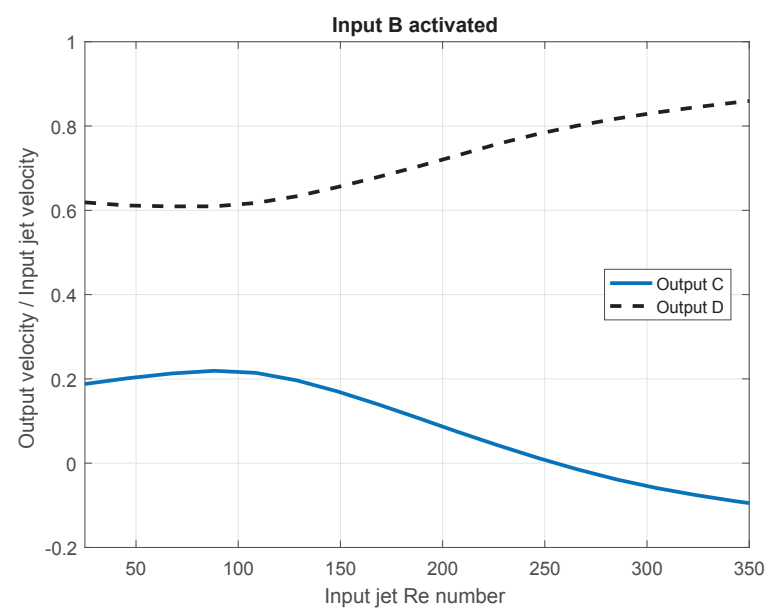

(c)

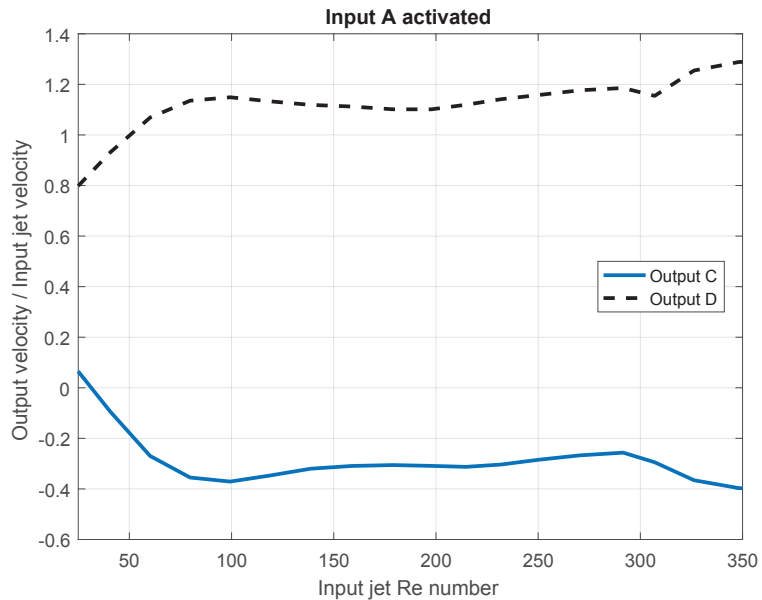

(b)

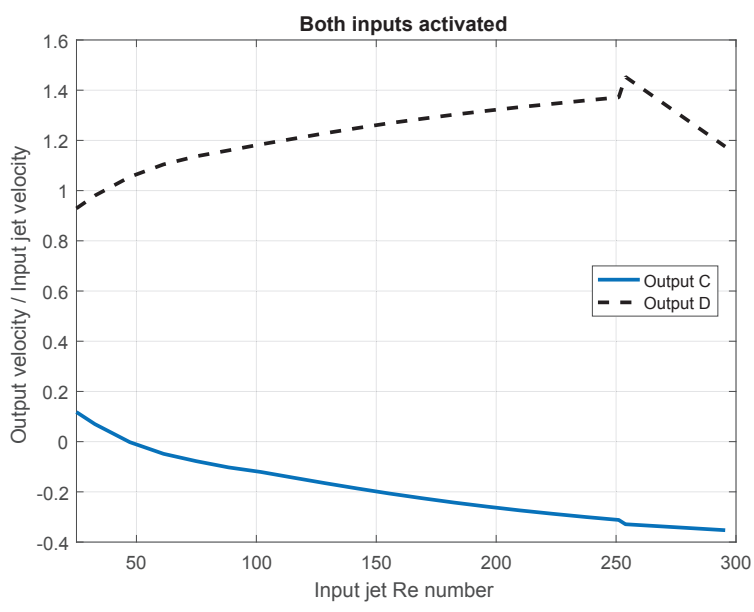

(d)

Figure 6: Relative velocity of fluid flow in output channels of or gate (Fig. 2p) with (a) no inputs activated, (b) input A activated, (c) input B activated, (d) both inputs activated.

\subsection{Grid sensitivity}

To evaluate outputs of the model for varying grid sizes, the behaviour of AND gate was simulated with both inputs activated and various grid sizes: $100 \times 100,200 \times 200$ and $400 \times 400$ nodes; this allowed us to find the value of error induced by changes in the grid dimensions. The results are presented in Fig. 8 .

It is evident that the simulation outputs are quite similar for all the selected dimensions of grids. Specifically, the fluidic gate appears to be functional, in terms of high output fluid velocities compared with the input velocities, for $R e$ numbers higher than 50. Despite the small relative error between the grids (as presented in Fig. 8(d) where the relative error of the ratio of output $\mathrm{E}$ to input velocities is depicted), the functionality of the gate is identical. Note here that grid size of $100 \times 100$ nodes was selected for all the simulations of this study, based on the trade-off between accuracy and execution time. 


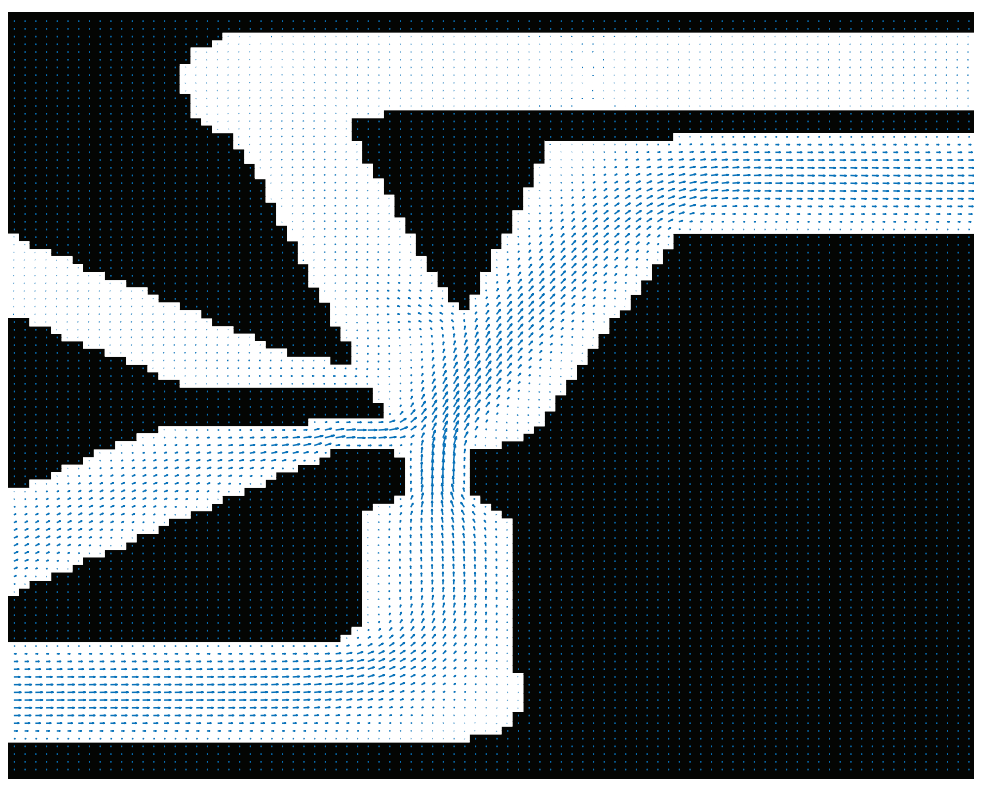

(a)

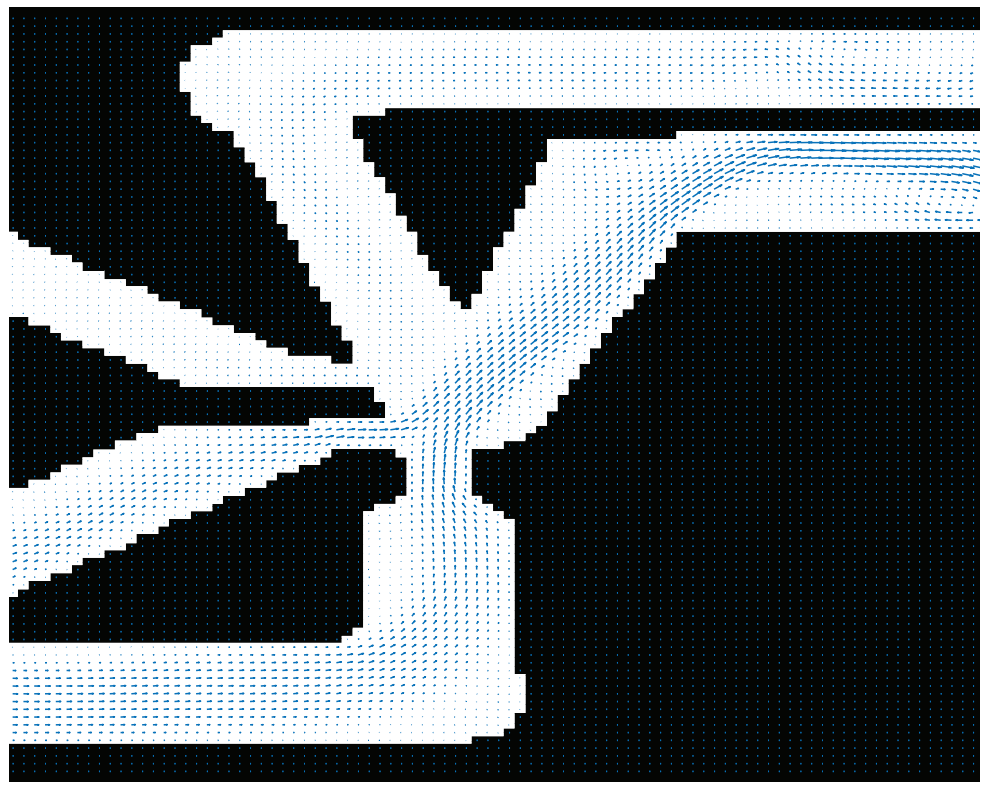

(c)

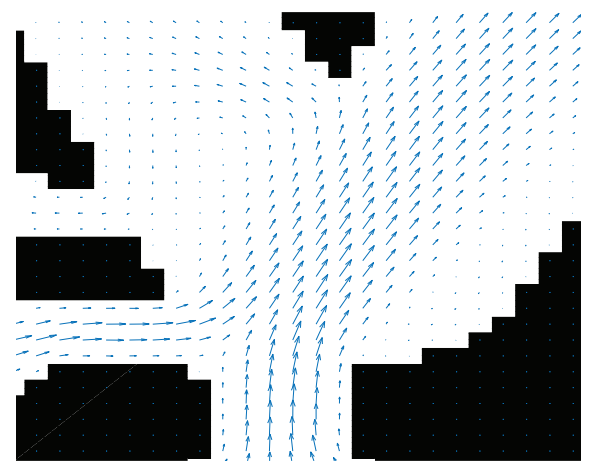

(b)

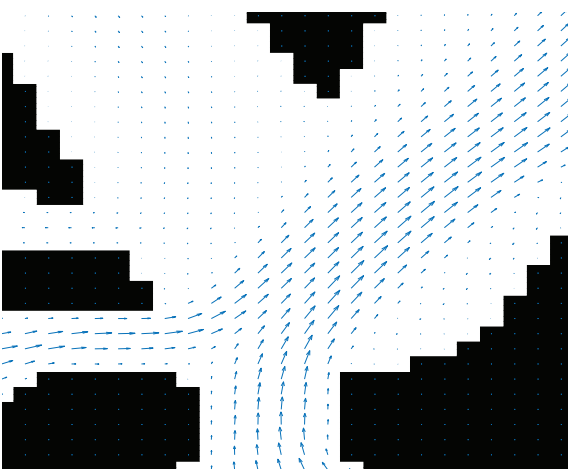

(d)

Figure 7: Flow field for OR gate (Fig. 2b) with input A activated for (ab) low, $R e=50$, and (cd) high, Re $=350$, Reynolds numbers. (ac) Overview of flow fields. (bd) Zoomed flow fields in the nozzle area. 


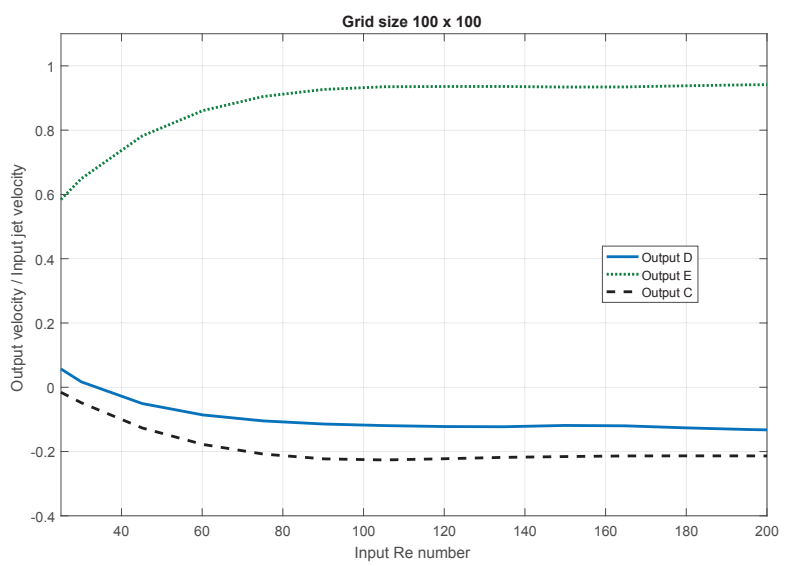

(a)

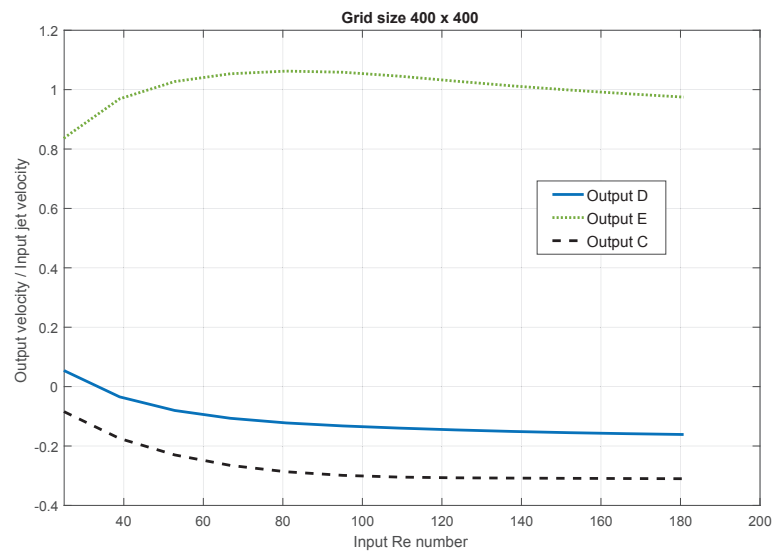

(c)

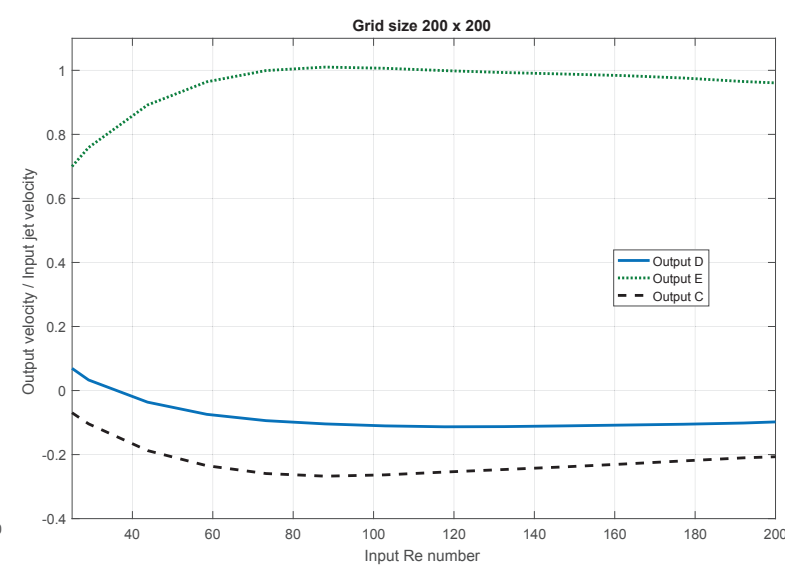

(b)

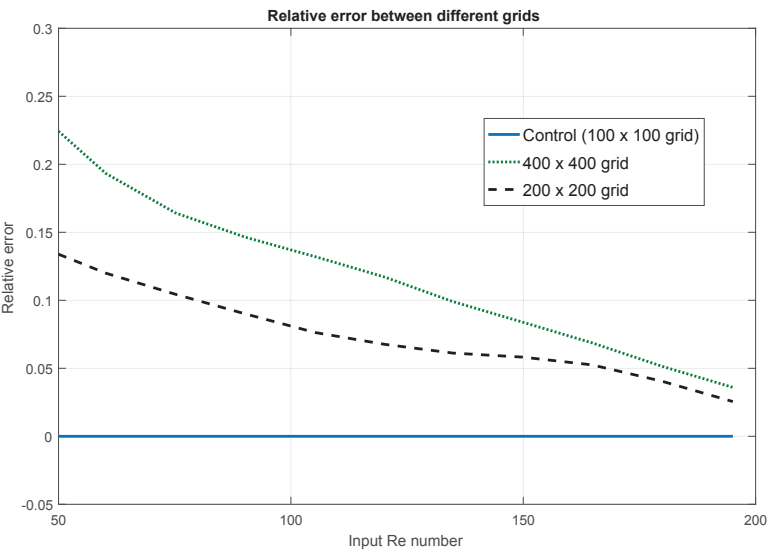

(d)

Figure 8: Outputs of the AND gate with both inputs simulated on a (a) $100 \times 100,(b) 200 \times 200$ and $($ c) $400 \times 400$ grid. (d) Relative error for different grids using the $100 \times 100$ grid as control for the ratio between output and input velocities.

\section{Discussion}

We simulated the operation of the fluidic logic gates AND and oR for fluid flow with Reynolds numbers ranging from 25 to 300 . To verify functioning of the gates we compared the velocity of fluid in output channels to that in the input channels. We found that AND gate efficiently functions for $R e \geq 100$ and oR gate for $R e \geq 300$. The higher threshold of the or gate is due to the high positioning of the nozzle of input B. A better configuration might result in a gate that would work properly for all possible input combinations with input flows of lower Re number.

Despite the fact that the results presented here refer to $2 \mathrm{D}$ cases, the extrapolation of LBM in $3 \mathrm{D}$ and the representation of the respective boundary geometries are straightforward. The 2D option was selected mainly for illustrative purposes and due to faster execution of the algorithm without a substantial decrease in accuracy.

The output results of the gates under flows of lower $R e$ numbers imply that the fluidic gates can still be used under the scope of fuzzy logic or approximate computing. In further studies we plan to cascade the gates into binary arithmetical circuits and study sensitivity of the circuits to $R e$ number. We will be searching for a possibility to make polymorphic fluidic gates, re-configurable by values of $R e$.

Fluidic devices could not replace well-established electronic circuits/gates in all possible systems. How- 
ever, the application of hybrid systems - accommodating electronic and fluidic circuits - could be more efficient in specific areas of scientific computation. A similar concept of hybrid systems, with both analog and digital signals, is implemented and proved to be efficient in specific applications 42 .

The motivation of this work is based on the fact that fluidic devices have been vastly utilized in control applications, due to their capacity to retain high efficiency in adverse environments, whereas being relatively inexpensive and of small size. Examples include gas chromatography [43, 44, respirator control [45, 46], fuel control [47, automotive cruise control [48, vehicle braking system [49, emission control [50, control of molding machines [51]. When properly maintained the fluidic devices are not affected by vibration, exposure to radiation and extreme temperatures. Nonetheless, fluidics are less prone to deterioration compared with other media.

Moreover, the concept of using fluidic gates can be targeting application domains where high velocity fluid flows are readily available, in order to result to free energy computations. For example, they can be utilized in fast moving vehicles, like airplanes [52, 53] or boats [54], or tall buildings, where dimensions are not limiting and water dropping from a considerable height can reach high velocities, thus, high $R e$ numbers.

\section{References}

[1] J. W. Joyce, Fluidics: basic components and applications, Tech. rep., Harry Diamond Labs Adelphi MD (1983).

[2] T. Nikola, Valvular conduit, uS Patent 1,329,559 (Feb. 3 1920).

[3] J. M. Kirshner, S. Katz, Design theory of fluidic components, Academic Press, 1975.

[4] M. J. Moylan, Fluid logic in simple terms, The Machinery Publishing Co. Ltd., 1968.

[5] A. Conway, Guide to Fluidics, Macdonald \& Cp. (Publishers) Ltd, 1971.

[6] R. W. Warren, B. M. Horton, Fluid logic components, uS Patent 3,107,850 (Oct. 22 1963).

[7] T. M. Conte, E. P. DeBenedictis, P. A. Gargini, E. Track, Rebooting computing: The road ahead, Computer 50 (1) (2017) $20-29$.

[8] J. M. Shalf, R. Leland, Computing beyond moore's law, Computer 48 (12) (2015) 14-23.

[9] Y.-H. Lu, A. M. Kadin, A. C. Berg, T. M. Conte, E. P. DeBenedictis, R. Garg, G. Gingade, B. Hoang, Y. Huang, B. Li, et al., Rebooting computing and low-power image recognition challenge, in: Computer-Aided Design (ICCAD), 2015 IEEE/ACM International Conference on, IEEE, 2015, pp. 927-932.

[10] J. Hasler, Opportunities in physical computing driven by analog realization, in: Rebooting Computing (ICRC), IEEE International Conference on, IEEE, 2016, pp. 1-8.

[11] A. Adamatzky (Ed.), Advances in Unconventional Computing. Volume 2: Prototypes, Springer, 2016.

[12] J. W. Joyce, Design guide for fluidic laminar proportional amplifiers and laminar jet angular rate sensors, Tech. rep., Harry Diamond Labs Adelphi MD (1984).

[13] H. A. Stone, S. Kim, Microfluidics: basic issues, applications, and challenges, AIChE Journal 47 (6) (2001) $1250-1254$.

[14] F. Battaglia, S. J. Tavener, A. K. Kulkarni, C. L. Merkle, Bifurcation of low reynolds number flows in symmetric channels, AIAA journal 35 (1) (1997) 99-105.

[15] J. U. Lee, J.-S. Hong, H.-G. Sung, Operation limit of flow control for a bistable fluidic valve, International Journal of Aeronautical and Space Sciences 18 (3) (2017) 389-394.

[16] V. Samsonov, E. Kurkin, O. Lukyanov, V. Shakhov, Fluidic logic element performance calculation, in: Applications of Fluid Dynamics, Springer, 2018, pp. 305-314.

[17] G. R. McNamara, G. Zanetti, Use of the boltzmann equation to simulate lattice-gas automata, Phys. Rev. Lett. 61 (1988) 2332-2335. doi:10.1103/PhysRevLett.61.2332

[18] F. J. Higuera, J. Jimnez, Boltzmann approach to lattice gas simulations, EPL (Europhysics Letters) 9 (7) (1989) 663.

[19] F. J. Higuera, S. Succi, R. Benzi, Lattice gas dynamics with enhanced collisions, EPL (Europhysics Letters) 9 (4) (1989) 345 .

[20] F. J. Higuera, S. Succi, Simulating the flow around a circular cylinder with a lattice boltzmann equation, EPL (Europhysics Letters) 8 (6) (1989) 517.

[21] S. Chen, G. D. Doolen, Lattice boltzmann method for fluid flows, Annual review of fluid mechanics 30 (1) (1998) 329-364.

[22] X. He, L.-S. Luo, Theory of the lattice boltzmann method: From the boltzmann equation to the lattice boltzmann equation, Physical Review E 56 (6) (1997) 6811.

[23] A. A. Mohamad, Lattice Boltzmann method: fundamentals and engineering applications with computer codes, Springer Science \& Business Media, 2011.

[24] B. Chopard, P. O. Luthi, Lattice boltzmann computations and applications to physics, Theoretical Computer Science 217 (1) (1999) 115-130.

[25] S. Reijers, H. Gelderblom, F. Toschi, Axisymmetric multiphase lattice boltzmann method for generic equations of state, Journal of Computational Science 17 (2016) $309-314$.

[26] H. Otomo, H. Fan, Y. Li, M. Dressler, I. Staroselsky, R. Zhang, H. Chen, Studies of accurate multi-component lattice boltzmann models on benchmark cases required for engineering applications, Journal of Computational Science 17 (2016) $334-339$. 
[27] G. Amati, S. Succi, R. Benzi, Turbulent channel flow simulations using a coarse-grained extension of the lattice boltzmann method, Fluid Dynamics Research 19 (5) (1997) 289.

[28] M. Bernaschi, S. Melchionna, S. Succi, M. Fyta, E. Kaxiras, J. Sircar, Muphy: A parallel multi physics/scale code for high performance bio-fluidic simulations, Computer Physics Communications 180 (9) (2009) 1495 - 1502. doi:https: //doi.org/10.1016/j.cpc.2009.04.001

[29] M. Bernaschi, M. Fatica, S. Melchionna, S. Succi, E. Kaxiras, A flexible high-performance lattice boltzmann gpu code for the simulations of fluid flows in complex geometries, Concurrency and Computation: Practice and Experience 22 (1) (2010) 1-14.

[30] P. Valero-Lara, F. D. Igual, M. Prieto-Matas, A. Pinelli, J. Favier, Accelerating fluidsolid simulations (Lattice-Boltzmann \& Immersed-Boundary) on heterogeneous architectures, Journal of Computational Science 10 (2015) $249-261$.

[31] D. Bartuschat, U. Rde, Parallel multiphysics simulations of charged particles in microfluidic flows, Journal of Computational Science 8 (2015) $1-19$.

[32] L. Mountrakis, E. Lorenz, O. Malaspinas, S. Alowayyed, B. Chopard, A. G. Hoekstra, Parallel performance of an IB-LBM suspension simulation framework, Journal of Computational Science 9 (2015) $45-50$.

[33] D. d'Humieres, Generalized lattice-boltzmann equations, Progress in Astronautics and Aeronautics 159 (1994) $450-450$.

[34] P. Lallemand, L.-S. Luo, Theory of the lattice boltzmann method: Dispersion, dissipation, isotropy, galilean invariance, and stability, Physical Review E 61 (6) (2000) 6546.

[35] C. Zhang, Y. Cheng, S. Huang, J. Wu, Improving the stability of the multiple-relaxation-time lattice boltzmann method by a viscosity counteracting approach, Advances in Applied Mathematics and Mechanics 8 (1) (2016) $37-51$.

[36] B. Chopard, A. Dupuis, A. Masselot, P. Luthi, Cellular automata and lattice boltzmann techniques: An approach to model and simulate complex systems, Advances in complex systems 5 (02n03) (2002) 103-246.

[37] Q. Zou, X. He, On pressure and velocity boundary conditions for the lattice boltzmann bgk model, Physics of fluids 9 (6) (1997) 1591-1598.

[38] T. J. P. Weathers, Nasa contributions to fluidic systems, Journal of Spacecraft and Rockets 10 (7) (1973) 417-418.

[39] E. Hobbs, Monograph in DOFL/HDL series on Fluid Amplification, Tech. rep., TR-1114. 9. "Logic Elements", 8 March 1963, 29 pp, Unclassified. DDC No. AD401-321. OTS No. Not available at OTS (1963).

[40] B. Newman, The deflection of plane jets by adjacent boundariescoanda effect, Boundary layer and flow control 1 (1961) $232-264$.

[41] I. Reba, Applications of the coanda effect, Scientific American 214 (6) (1966) 84-93.

[42] S. Sethumadhavan, R. Roberts, Y. Tsividis, A case for hybrid discrete-continuous architectures, IEEE Computer Architecture Letters 11 (1) (2012) 1-4.

[43] R. L. Wade, S. P. Cram, Fluidic logic sampling and injection system for gas chromatography, Analytical Chemistry 44 (1) (1972) 131-139.

[44] R. Annino, J. Leone, The use of coanda wall attachment fluidic switches as gas chromatographic valves, Journal of Chromatographic Science 20 (1) (1982) 19-26.

[45] W. Lederman, A. Pawlak, Fluidic respirator control system with patient triggering response means, uS Patent 3,834,382 (September 1974).

[46] M. Klain, R. Smith, Fluidic technology, Anaesthesia 31 (6) (1976) 750-757.

[47] J. M. Lazar, Fluidic fuel control system, uS Patent 3,463,176 (August 1969).

[48] C. K. Mcconnell, Automotive cruise control system having fluidic control devices, uS Patent 3,578,108 (May 1971).

[49] E. T. Dressler Jr, Fluidic control system for vehicle brakes, uS Patent 3,606,484 (September 1971).

[50] W. F. Gesell, Emission control system, uS Patent 4,094,284 (June 1978).

[51] F. H. Blake, Processed-paced, fluidic control system for molding machines, uS Patent 3,998,574 (December 1976).

[52] H. D. Garner, Applications of fluidics to light aircraft instrumentation and control, Tech. rep., SAE Technical Paper (1974).

[53] P. Prohaska, Stabilizing system, uS Patent 3,727,573 (April 1973).

[54] B. A. Jones, Fluid amplification device for propulsion system roll control, uS Patent 3,229,461 (January 1966). 